\title{
Scalar dissipation rate and scales in swirling turbulent premixed flames
}

M. Mustafa Kamal ${ }^{\mathrm{a}, \mathrm{c} *}$, Bruno Coriton ${ }^{\mathrm{b}}$, Ruigang Zhou ${ }^{\mathrm{a}}$, Jonathan H. Frank ${ }^{\mathrm{b}}$, Simone Hochgreb ${ }^{\mathrm{a}}$

${ }^{a}$ Department of Engineering, University of Cambridge, CB2 1PZ, Cambridge, UK

${ }^{\mathrm{b}}$ Combustion Research Facility, Sandia National Laboratories, Livermore, CA 94551, USA

${ }^{c}$ Department of Mechanical Engineering, University of Engineering and Technology, Peshawar, Pakistan

Corresponding author: M. Mustafa Kamal

Department of Engineering, University of Cambridge

Cambridge, CB2 1PZ, United Kingdom

Fax: (+44) (0) 1223764311

Email: mustafa.kamal@cantab.net

Colloquium topic: 5 .

Turbulent flames

Alternative Colloquium topic: 3. Diagnostics

\section{Paper length (method 1):}

$\begin{array}{lll}\text { Main Text: } & \text { word processor count } & =3538 \\ \text { Equations: } & (0+0) \times(7.6 \text { words/line }) \times(1 \text { columns }) & =0 \\ \text { References: } & (35+2) \times(2.3 \text { lines } / \text { reference }) \times(7.6 \text { words } / \text { line }) & =623 \\ \text { Tables: } & (7+4) \times(7.6 \text { words } / \text { line }) \times(1 \text { columns }) & =84\end{array}$

Figures: Word count $=($ figure height in $\mathrm{mm}+10 \mathrm{~mm}) \times(2.2$ words $/ \mathrm{mm}) \times(\#$ columns $)+(\#$ words in caption $)$

Figure 1: $\quad(60 \mathrm{~mm}+10) \times(2.2$ words $/ \mathrm{mm}) \times(1$ column $)+24 \quad=178$

Figure 2: $\quad(33 \mathrm{~mm}+10) \times(2.2$ words $/ \mathrm{mm}) \times(1$ column $)+38 \quad=132$

Figure 3: $\quad(139 \mathrm{~mm}+10) \times(2.2$ words $/ \mathrm{mm}) \times(1$ column $)+41 \quad=368$

Figure 4: $\quad(71 \mathrm{~mm}+10) \times(2.2$ words $/ \mathrm{mm}) \times(1$ column $)+33 \quad=211$

Figure 5: $\quad(104 \mathrm{~mm}+10) \times(2.2$ words $/ \mathrm{mm}) \times(1$ column $)+56 \quad=306$

Figure 6: $\quad(79 \mathrm{~mm}+10) \times(2.2$ words $/ \mathrm{mm}) \times(1$ column $)+14=209$

${ }^{*}$ Corresponding author

Email address: mustafa.kamal@cantab.net 
Figure 7: $\quad(104 \mathrm{~mm}+10) \times(2.2$ words $/ \mathrm{mm}) \times(1$ column $)+10 \quad=260$

Figure 8: $\quad(115 \mathrm{~mm}+10) \times(2.2$ words $/ \mathrm{mm}) \times(1$ column $)+57=333$

Total figures: 1965

Total $=6242$ words

Color reproduction: online only (color figures are to be printed in gray scale) 


\title{
Scalar dissipation rate and scales in swirling turbulent premixed flames
}

\author{
M. Mustafa Kamal ${ }^{\mathrm{a}, \mathrm{c} *}$, Bruno Coriton ${ }^{\mathrm{b}}$, Ruigang Zhou ${ }^{\mathrm{a}}$, Jonathan H. Frank ${ }^{\mathrm{b}}$, Simone Hochgreb ${ }^{\mathrm{a}}$ \\ ${ }^{a}$ Department of Engineering, University of Cambridge, CB2 1PZ, Cambridge, UK \\ ${ }^{\mathrm{b}}$ Combustion Research Facility, Sandia National Laboratories, Livermore, CA 94551, USA \\ ${ }^{c}$ Department of Mechanical Engineering, University of Engineering and Technology, Peshawar, Pakistan
}

\begin{abstract}
Simultaneous Rayleigh scattering and OH-PLIF imaging measurements of temperature and $\mathrm{OH}$ were used to investigate the properties of turbulent premixed flames, including the nature of the 2D thermal structures and scalar dissipation rate in the Cambridge/Sandia swirling bluff body stabilized flames, with and without the effect of swirl. Swirl creates enhanced turbulence as well as outer flow entrainment, and disrupts the pre-flame zone significantly, whilst the high temperature reaction zone as marked by $\mathrm{OH}$ remains relatively intact. In particular, the temperature at the location of maximum $\mathrm{OH}$ gradient shows very low variance across the flame region.
\end{abstract}

The 2D image analysis of $\mathrm{OH}$ and temperature shows that the corresponding $2 \mathrm{D}$ gradients are aligned up to a distance of half the laminar flame thickness away from the flame front, deviating significantly in the case of swirling flames beyond that region. As in previous investigations in diffusion flames, the mean width of the observed thermal structures increases from 300 to 600 microns near the flame, with a main mode around the laminar flame thermal width in the unswirled case. The correlation between 2D thermal fluctuation gradients and variance extracted from the images shows a direct proportionality, with a slope which agrees well with theory in the region of high turbulence away from the base. At the

${ }^{*}$ Corresponding author

Email address: mustafa.kamal@cantab.net 
base of the flame where turbulence is low, the local scalar dissipation becomes a function of the local temperature via the thermal diffusivity.

Keywords: Turbulent premixed flame; Scalar dissipation rate; Dissipative structures; Swirl burner; 2D Rayleigh scattering 


\section{Introduction}

The rate of turbulent micromixing between hot products and reactants controls the overall rate of heat release per unit volume in a flame [1] [2]. In the limit of fast reactions at high Damköhler number $(\mathrm{Da})$, the reaction rate becomes proportional to the scalar dissipation rate, and the flame thickness becomes much smaller than the turbulent spatial scales. Under this thin flame regime, the assumption is often made that the reaction region remains intact, while the reacting interface is transported and extended by turbulence; the reaction rate becomes proportional to the rate at which the flame front propagates through the mixture. This is expressed via the flame surface density [1] [3] [4], or via the rate of scalar dissipation, characterized by the rate of micromixing of the relevant scalar fluctuation [5] [6] [7] [8]. The mean Favre-averaged scalar dissipation rate of a progress of reaction $c$, is denoted as $\tilde{\chi}_{c}=\overline{\rho D \nabla c^{\prime \prime} \nabla c "} / \bar{\rho}$, where $\rho$ and $D$ are the mixture density and scalar diffusivity, and the usual notation is used: bars for average, tilde for density averaged and double primes for turbulent fluctuation away from the local Favre mean.

Many previous experimental studies have explored the internal structure of premixed turbulent flames, to understand under what conditions the reaction zone structure may be affected by turbulence. A number of studies measured a thickening of the preheating zone (also known as thermal or pre-flame zone) due to enhanced transport caused by structures smaller than the preheating zone [9] [10] [11] [12], whereas others [13] [14] suggest a thinning effect. In references [13] [14], simultaneous OH planar laser-induced fluorescence (PLIF) and Rayleigh scattering imaging were used to show that for Karlovitz (Ka) numbers up to 20 , the reaction zone is undisturbed; subsequent studies showed that much higher values are required to disrupt the flame zone [15].

Direct numerical simulations of high Ka turbulent flames by Aspden et al. [16] and Poludnenko and Oran [17] show that although the pre-flame zone is significantly disrupted, 
the reaction zone remains rather undisturbed, and that the main effect of turbulence is the wrinkling of the reaction zone. A number of experiments have attempted to connect the effects of turbulence intensity and integral length scale to the behaviour of surrogate scalars, showing for example a widening distribution of intermediates such as formaldehyde with increasing turbulence [1] [18] [19] [20] [21] [22]. A full description of the interaction between turbulence and reaction rate remains a key problem in combustion.

Previously, Chen and Bilger [12] combined single-plane measurements of $\mathrm{OH}$ and twoplane measurements of temperature to study the structure of turbulent methane, propane and hydrogen Bunsen flames, for Karlovitz numbers of up to 3 in the hydrocarbon flames and 10 in hydrogen flames. These measurements quantified the gradients of temperature, scalar dissipation and a number of statistical properties of these Bunsen flames [12]. Frank and Kaiser used similar techniques with higher resolution [23] [24] for measurements of thermal dissipation rate and length scales in diffusion flames. Wang et al. [25] [26] analysed the resolution requirements for separating noise from signal in scalar dissipation measurements.

The present measurements are based on the same techniques developed by Frank and Kaiser [23] [24] for measurements of thermal dissipation rate and corresponding length scales in diffusion flames. The present imaging measurements address the scalar structures in the swirling, bluff-body stabilized methane V-flames extensively described by Sweeney et al. [11], at Ka numbers up to 7 . These complement previous measurements in turbulent premixed and stratified flames by Sweeney et al. [11] [27] [28] using line Raman/Rayleigh/CO-LIF and cross-planar OH-PLIF techniques. The latter provided detailed information on local concentrations of major species and temperature, as well as the local temperature gradients at the flame along a line, and corresponding 3D gradients at the flame front. However, such line measurements cannot offer insight into the detailed structure of mixing and scalar dissipation rate in the flow. 
In the present work, we use high resolution Rayleigh scattering and OH LIF imaging to measure the $2 \mathrm{D}$ length scales of the characteristic structures, as well as the $2 \mathrm{D}$ thermal dissipation rate, for the same premixed flames at no swirl and high swirl conditions. The analysis also examines the assumption of alignment between the temperature and $\mathrm{OH}$ gradient, used in previous papers [11], and the robustness of the relationship between dissipation rate and variance suggested by turbulent combustion models [6] [29] [30].

\section{Experimental details}

\subsection{Cambridge/Sandia stratified swirl burner and operating conditions}

The geometry of the swirl burner is described in detail in Ref. [28]. Long co-annular tubes ensure that a well-developed turbulent flow is delivered to the flame region. A ceramic central bluff body is used to stabilize the flame with minimal heat loss. Flow through the outer annulus is split between an axial and a swirling flow emerging at the base of the burner, providing a maximum swirl flow ratio (SFR) of 0.33 [28].

Imaging measurements were performed at the three locations indicated by the field of view (FOV) regions in Fig. 1. Each field of view is approximately centered at the local midpoint of the flame brush, with dimensions of $24.5 \times 16.3 \mathrm{~mm}^{2}$ and centreline coordinates as listed in Table 1. A subset of the previously used operating conditions in Ref. [28] is studied in this paper, focusing on comparisons between non-swirling (SFR=0\%) and highly swirling $(\mathrm{SFR}=33 \%)$ flames, with no stratification between the inner and outer annuli, with a premixed equivalence ratio of $\phi=0.75$ (Table 2).

\begin{tabular}{|c|c|c|c|c|c|}
\hline Case & SFR (\%) & S (-) & FOV 1 & FOV 2 & FOV 3 \\
\hline SwB1 & 0 & 0.03 & $(05,15)$ & $(08,30)$ & $(11,50)$ \\
\hline $\boldsymbol{S w B 3}$ & 33 & 0.54 & $(13,15)$ & $(21,30)$ & $(27,50)$ \\
\hline
\end{tabular}


Table 1. Operating conditions for the flames considered. The velocity of the inner channel is $U_{i}=8.3$ $\mathrm{m} / \mathrm{s}\left(\operatorname{Re}_{\mathrm{i}}=5960\right)$ and for the outer channel, $U_{o}=18.7 \mathrm{~m} / \mathrm{s},\left(\operatorname{Re}_{\mathrm{i}}=11500\right.$ based on hydraulic diameter of the outer annulus) with co-flow velocity $U_{c o}=0.4 \mathrm{~m} / \mathrm{s}$. Coordinates of the center of the FOV in radial and axial distances from the centreline at the base of the burner, in mm. SFR: ratio of incoming tangential to total flow rate.

\begin{tabular}{|l|c|c|c|c|}
\hline \multirow{4}{*}{ Flames } & \multicolumn{4}{|c|}{$\frac{\boldsymbol{U}^{\prime}}{\boldsymbol{S}_{\boldsymbol{L}}}$} \\
& \multicolumn{5}{|c|}{} \\
\hline $\mathbf{z}(\mathbf{m m})$ & $\mathbf{2}$ & $\mathbf{1 0}$ & $\mathbf{3 0}$ & $\mathbf{5 0}$ \\
\hline SwB1 & 11.5 & 9.7 & 10.0 & 9.8 \\
\hline SwB3 & 31.8 & 27.5 & 17.9 & 12.9 \\
\hline
\end{tabular}

Table 2. Maximum value of the ratio for the total mean velocity fluctuation $U^{\prime}$ divided by the laminar speed $S_{L}$ for a given axial location, $z$. Turbulent velocity measurements acquired with laser Doppler anemometry (LDA) and particle image velocimetry (PIV) by Zhou et al. [28].

\subsection{Optical arrangement}

Simultaneous Rayleigh scattering and OH-PLIF imaging measurements were performed in the Advanced Imaging Laboratory at Sandia National Laboratories. The experimental arrangement consisted of the high-resolution Rayleigh imaging apparatus described in Ref. [31], combined with an additional laser/camera system for simultaneous OH-PLIF imaging [23].

A laser sheet (100 $\mu \mathrm{m}$ thickness) used for Rayleigh scattering was produced from a frequency-doubled Nd:YAG laser, with an energy of $2.0 \mathrm{~J} /$ pulse at $532 \mathrm{~nm}$ at a repetition rate of $10 \mathrm{~Hz}$. OH PLIF was excited by the frequency-doubled output of a 10-Hz Nd:YAGpumped dye-laser tuned to $283.01 \mathrm{~nm}$ to pump the $\mathrm{Q}_{1}(6)$ transition of the $\mathrm{A}-\mathrm{X}(1,0)$ band of $\mathrm{OH}$ using $1.2 \mathrm{~mJ} / \mathrm{pulse}$.

Rayleigh scattering from the probe volume was imaged onto an unintensified interlinetransfer CCD camera (SensiCam QE, PCO/Cooke). A second interline-transfer camera was 
used to image Rayleigh scattering from the air co-flow adjacent to the burner to account for shot-to-shot variations in the laser profile. The Rayleigh scattering from room temperature air was used as a reference signal. The background signal was extracted from measurements in pure air and helium streams which have known Rayleigh scattering cross sections.

The expanded laser beams used for Rayleigh scattering and OH-PLIF were overlapped by stacking the beam planes crossing the centreline of the burner along the axial direction. The $\mathrm{OH}$ fluorescence emission was imaged onto an intensified CCD camera (Andor DH312T$18 \mathrm{H}-83)$. The shot-to-shot laser energy fluctuations of $\pm 2 \%$ were negligible for the subsection of the dye-laser beam that was used for PLIF imaging. The OH-PLIF images were corrected for variations in the average dye-laser beam profile. The FOV of the Rayleigh camera was a $19.3 \times 14.6 \mathrm{~mm}^{2}$ subsection of the $24.5 \times 16.3 \mathrm{~mm}^{2}$ region imaged by the OH-PLIF camera. The pixel resolution of the Rayleigh scattering measurement was $14 \mu \mathrm{m} /$ pixel. The resolution of the OH-PLIF camera was approximately $140 \mu \mathrm{m} / \mathrm{pixel}$ and was limited by the resolution of the image intensifier.

Both OH PLIF images and Rayleigh images were smoothed using a Gaussian filter, with the latter relying on a variable, temperature-dependent kernel as described in [31] [24]. Additional information regarding the image processing and system setup are detailed in the relevant references. A fixed Rayleigh scattering cross section equal to 1.09 times that of air was used to determine the temperature for all conditions in Table 1. The maximum uncertainty in the temperature measurement resulting from composition variations was estimated as $\pm 3-4 \%$. 


\section{Data analysis}

Figures $2 \mathrm{a}$ and $2 \mathrm{~b}$ show an example of normalized $\mathrm{OH}$ LIF signal and the temperature obtained from the Rayleigh scattering signal, respectively. The flame front location is defined here as the location of the maximum gradient of the OH PLIF signal [32], and is indicated by a black line in the figure. For the present comparisons, this definition provides a well defined reference that is expected to be near the location of peak heat release rate [32]. The local flame normal is defined with respect to the flame front contour, and is shown as white dashed lines in the figure.

Local ensemble averaged temperatures $\langle T\rangle$ and the corresponding root mean squared temperature fluctuations $\left\langle T^{\prime}\right\rangle$ are obtained from 2000 individual shots for each FOV. A local flame temperature $T_{f}$ is defined as the instantaneous temperature at the flame front. Deviations of temperature relative to the instantaneous local flame temperature are defined along the normal coordinate $n$, as $T_{f}^{\prime}=T-T_{f}$, with corresponding mean and rms values, $<T_{f}>$ and $<T_{f}^{\prime}>$, respectively.

The 2D temperature gradients $\nabla T=\frac{d T}{d r} \boldsymbol{i}+\frac{d T}{d z} \boldsymbol{k}$ are determined via first order central differencing after filtering. The gradients of the temperature fluctuations are defined as $\nabla T^{\prime}=\nabla(T-<T>)$. In previous line measurements [11], the 3D temperature gradients throughout the flame were assumed to be parallel to the flame normal obtained from the cross planar OH PLIF measurements. Here we assess the quality of this assumption in $2 \mathrm{D}$ by determining the statistics of the angle $\theta$ between the temperature and $\mathrm{OH}$ gradients as a function of temperature. Further, the characteristic $2 \mathrm{D}$ thermal diffusion width, $\lambda_{D}$, of the dissipation layer is obtained from the images. The diffusion width is defined as the full width at $20 \%$ of the local maximum temperature gradient, as determined along the direction normal to the layer, which provides a more direct measure of the structure length scale than is possible with 1D measurements [24]. The value of the thermal diffusivity, $\alpha$, is obtained by 
interpolating a lookup table of calculated values as a function of temperature from premixed unstrained flames at premixed conditions of $\phi=0.75$.

\section{Results and discussion}

\subsection{Single shot measurements}

Single shot images of OH PLIF signal, temperature and respective fluctuations for both flow conditions at the regions nearest to the base, FOV1 (bottom panel), and furthest away, FOV3 (top panel), are shown in Fig. 3. For the non-swirling case, SwB1, a comparison of the temperature and OH PLIF images shows that the overall topologies of the OH-PLIF images are well aligned with the temperature images, even in the furthest downstream regions where turbulence is more developed. The flame front contour (location of the highest gradient $\mathrm{OH}$ signal) overlaps almost exactly with an isotherm at approximately $1450 \mathrm{~K}$ throughout the flame region, with a thermal thickness that is only slightly larger than the laminar flame thickness of $\delta_{L}=588 \mu \mathrm{m}$ calculated for $\phi=0.75$.

Contrast this behavior with the swirling case, SwB3 (Fig. 3, right), in which the preheating region is highly disrupted and convoluted, particularly in the region further downstream (FOV3): regions of high temperature appear in the reactant region, which could be a result of out-of-plane motion of neighboring regions of the flame, as well as entrainment of the flame structures into the reaction zone. The product temperatures are lower than in SwB1 as a result of the swirl-induced entrainment of co-flow air into the central product zone [11] [28]. The OH PLIF signal boundary and the corresponding flame contour is significantly distorted but not dispersed throughout the pre-heating zone. Although the $\mathrm{OH}$ radical is not a definitive marker of the flame location under all conditions, the distribution of the OH PLIF 
signal is very different from that of the temperature. For temperatures above $1200 \mathrm{~K}$, there are no clearly detectable thermal structures.

The third row in each panel of Fig. 3 shows the instantaneous fluctuation, $T^{\prime}$, relative to the local mean temperature for the two regions FOV 1 and FOV 3. The highest deviations from the mean temperature straddle the location of the flame, as expected.

\subsection{Flame temperature statistics}

Figure 4 shows ensemble mean temperatures and rms fluctuations along the coordinate normal to the flame, $n$, where $n=0$ is the flame front defined as the location of maximum gradient in the $\mathrm{OH}$ LIF signal. The variance is lowest at $n=0$, increases to a maximum away from the flame front, and eventually decreases to zero in the products and reactants. Laminar unstrained premixed flame simulations at $\phi=0.75$ (based on GRI Mech 3.0 [33] and full transport properties) are also shown. The measured maximum conditional mean temperatures in the present dataset appear to be systematically lower than the calculated adiabatic flame temperature (which agrees well with the line measurements in [11]), by $150 \mathrm{~K}$ in the nonswirl case. The sources of the discrepancies are unclear, as all calibrations and procedures have been checked.

The mean product temperature in the swirling case $\mathrm{SwB} 3$ is lower than the corresponding non-swirling case SwB1 owing to entrainment. The fluctuation in temperature is significantly increased by the addition of swirl, particularly in the pre-flame region, due to entrainment of air, both around the outer edges of the flame, as well as through the top in the open recirculation zone [11] [28]. The low fluctuation in temperature at the flame front shows that the location of peak $\mathrm{OH}$ gradient, and presumably the reaction zone, is tied to a particular temperature, indicating that the flames appear as curved and strained flamelets. 


\subsection{Temperature and $\mathrm{OH} 2 \mathrm{D}$ gradient alignment}

Since temperature and $\mathrm{OH}$ are the most easily imaged scalars, these are often used as surrogates for progress of reaction markers [13] [14] [27] [34]. However, it is clear that their behaviour in the present flames can be very different, and that isocontours of temperature do not always align with those of OH LIF signals. In the present study, it is possible to identify, in $2 \mathrm{D}$, how well the isoscalars align around the flame. In particular, in the study using line measurements [11], two-plane OH LIF imaging was used to determine the orientation of the 3D flame normal for the flame crossing the measurement line. This orientation was then used to correct the temperature gradient along the measurement line, under the assumption that the $\mathrm{OH}$ contour is parallel to the temperature contour in the vicinity of the thermal zone. Figure 5 shows how well this assumption holds by evaluating the angle between the normal directions for contours of temperature and OH LIF signal as a function of the flame distance from the flame along its normal divided by the laminar flame thickness, $n / \delta_{L}$. Near the flame front contour at $n / \delta_{L}=0, \theta$ is approximately zero, indicating that the temperature gradients are well aligned with the $\mathrm{OH}$ LIF gradients. At distances beyond $\delta_{L} / 2$, the assumption starts to break down as the angle increases.

\subsection{Thermal dissipation scales}

The propagation of reactions in a flame depends on the effective generation and dissipation of turbulent structures throughout the flame [5] [6], allowing the increase in the mean rate of reaction throughout the flame. The distribution and characteristic width of the structures generally follow that of a passive scalar, but are significantly affected by the local temperatures, which increase the diffusivity and viscosity, thus suppressing turbulence, and increasing length scales. This has been shown to be true in diffusion flames [23] [24]; here we demonstrate the similarities and differences for swirling premixed flames. 
Instantaneous images of the thermal dissipation structures are shown in Fig. 6: for the non-swirling case (left), the structures are associated primarily with the flame, and evolve slowly as the turbulence develops downstream. For the high swirl case, the structures are widely distributed, owing to the higher turbulence and entrainment of outside air. Yet these structures become thicker towards the product region (towards the right of each image).

In Figure 7, the dissipation structures are characterized by considering the probability density functions (pdf) of their width, $\lambda_{D}$, as a function of the temperature across the flames. The pdfs are conditioned to the temperature of the structure, so that the modes are not representative of the overall image, as the integral of each pdf in Fig. 7 is unity. In general, the structures become systematically thicker towards the high temperature regions, owing to the combined effects of dilatation and increasing viscosity with temperature. Beyond $1400 \mathrm{~K}$, detectable structure widths are mostly a result of the noise remaining from the beam steering correction. For the non-swirling flame $\mathrm{SwB} 1$, the most probable width of $600 \mu \mathrm{m}$ appears at $1000 \mathrm{~K}$ and corresponds closely to the unstrained laminar flame thickness of $588 \mu \mathrm{m}$. For the high swirl case SwB3, the PDF of layer widths has a peak around $300 \mu \mathrm{m}$ at $400 \mathrm{~K}$, arising from the mixing of product and fresh reactants via entrainment. Less frequent, thicker flame structures appear as temperature increases, to values approximating the width of the unstrained laminar flame, as in case SwB1. In case SwB3, the width of the PDF distributions increases with temperature, and the overall unconditioned PDF distribution is significantly broader than in the non-swirl case.

Turbulent combustion models rely on the relationship between statistical variances of the gradients of the reaction progress variable that represents micromixing and the average reaction rate. DNS studies have been used to scale and adjust models based on such correlations [5] [6] [7] [8]. Previous models for scalar dissipation suggest a linear correlation between the Favre mean scalar dissipation of the reaction progress variable, $\tilde{\chi}_{c}$, 
and the variance of the relevant progress variable, $\widetilde{c "}$. Specifically, the work by Kolla et al. [30] and references therein based on the original Bray model [35] suggest a specific correlation for the normalized Favre averaged scalar dissipation of a reaction progress variable to its variance, with a coefficient of order unity in the ideal case of high Da. Using temperature as the progress of reaction, normalized by $\Delta T$, the temperature rise for the adiabatic flame (1622 $\mathrm{K}$ for $\phi=0.75)$, it is possible to reproduce these quantities (Fig. 8) in the form of the normalized Favre averaged scalar dissipation $\hat{\chi}_{c}=\tilde{\chi}_{T} \tau_{f}=\frac{\tau_{f}}{\Delta T \bar{\rho}}<$ $\rho \alpha\left|\nabla T^{\prime \prime}\right|^{2}>$, where $\tau_{f}=\frac{\delta_{L}}{S_{L}}$ is the laminar flame transit time. The laminar flame transit time and the density were estimated from the temperature measurements, but variations in mixture composition were neglected. Values for $\hat{\chi}_{c}$ are plotted as a function of the Favre averaged normalized variance in temperature as progress of reaction, $\widetilde{c^{\prime \prime 2}}=\widetilde{T^{\prime 2}} / \Delta T^{2}=$ $\left\langle(\rho / \bar{\rho})(T-\tilde{T})^{2}\right\rangle / \Delta T^{2}$, and colored by the local mean temperature $\langle T\rangle$.

The plots in Fig. 8 for FOV1 and FOV2 of SwB1 show two branches, corresponding to the higher and lower temperature regions of the flame, reflecting the temperature dependence of the thermal diffusivity. Further away from the base, additional turbulence is generated by shear at the edges, the slope becomes lower, and the two branches merge. At this limit, the mean scalar dissipation rate becomes highly correlated with the mean variance in a nearly linear relationship, indicating that the variations in molecular diffusivity have less of an influence. The theory and DNS predictions for the relevant constant suggest that the slope of the curve should scale as [30]:

$\hat{\chi}_{c}=\frac{1}{\beta}\left[\left(2 \frac{K_{c}^{*}}{\theta_{T}}-C_{4}(1+K a)^{-0.4}\right) \theta_{T}+C_{3}\left(1+K a^{-0.5}\right)^{-1} D a^{-1}\right] \widetilde{c^{\pi 2}}$

where $\theta_{T}$ is the ratio of absolute product to reactant temperatures, $\mathrm{Da}=\tau_{T} / \tau_{f}$ is the Dahmköhler number and $\mathrm{Ka}=\tau_{f} / \tau_{\eta}$ the Karlovitz number, $\tau_{T}$ the integral turbulent time 
scales, $\tau_{\eta}$ the Kolmogorov time scale, and the remaining quantities are constants. The values of the constants proposed by Kolla et al. [30] are approximately $\frac{K_{c}^{*}}{\theta_{T}}=0.82$ to $0.85, C_{4}=$ 1.1, $C_{3}=1.1$ and $\beta=6.7$, and values for the present mixture are: $\theta_{T}=1+\frac{\Delta T}{T_{0}}=6.5$. In the limit of $\mathrm{Da}$ and zero Ka, the slope indicated by the model should be 0.53 to 0.58 . Higher values of turbulence would in general decrease Da and increase Ka. The measured slopes for SwB3 are closer to the limit model than those of SwB1, with the closest agreement at location FOV3. The higher turbulence levels in SwB3 and at FOV3 are consistent with the closer match to the theory. On the other hand, for $\mathrm{SwB} 3$ there is significant entrainment and mixing, so that one would expect a departure between the correlation between variance and dissipation. Further work connecting the present measurements to local turbulent velocity measurements should enlighten the correlation between theory, experiments and correlations extracted from DNS calculations.

\section{Conclusions}

The present investigation showed how swirl and the ensuing high turbulence and entrainment significantly disrupt the flame thermal zone in a premixed flame, whilst the high temperature reaction zone interface, as marked by the $\mathrm{OH}$ contour, remains relatively intact. The temperature at which the maximum $\mathrm{OH}$ gradient appears remains relatively unchanged, showing the lowest variance across the flame region, and coinciding with an isocontour of about $1450 \mathrm{~K}$. In support of previous assumptions regarding the alignment of $\mathrm{OH}$ and temperature contours, the present experiments showed that indeed, the $2 \mathrm{D}$ gradients of $\mathrm{OH}$ and temperature align well, up to a distance of about one half the laminar flame thickness away from the flame front, with significant deviations beyond that region in the case of swirling flames. 
Similarly to previous investigations of diffusion flames, characteristic diffusive widths of the observed thermal structures vary from 300 to 600 microns, from the non-reacting to the higher temperature regions, as a consequence of dilatation and increasing viscosity. The most probable structure widths coincide with the laminar flame thickness in the unswirled, whilst a more uniform distribution of widths appears in the latter case, with a peak at around $400 \mathrm{~K}$ temperatures, corresponding to mixing of product and fresh reactants.

The Favre-averaged 2D scalar dissipation obtained from the temperature gradients correlates well with the measured variance in the high turbulence regions. In the low turbulence region near the base, the value of the scalar dissipation is not monotonic with the variance of the progress variable and the local temperature. Future work should target understanding the details of these correlations based on the local turbulent velocity characteristics.

\section{Acknowledgements}

The Leverhulme Trust funded the collaboration through an International Network grant for Stratified Flames. This work at Sandia was supported by the U.S. Department of Energy, Office of Basic Energy Sciences, Division of Chemical Sciences, Geosciences, and Biosciences. Sandia National Laboratories is a multiprogram laboratory operated by Sandia Corporation, a Lockheed Martin Company, for the U.S. Department of Energy under contract DE-AC04-94-AL85000. 


\section{References}

[1] J. F. Driscoll, Prog. Energy Combust. Sci. 34 (2008) 91-134.

[2] K. N. C. Bray, P. A. Libby, and J. B. Moss, Combust. Flame 61 (1985) 87-102.

[3] I. G. Shepherd, Proc. Combust. Inst. 26 (1996) 373-379.

[4] Ö. L. Gülder and G. J. Smallwood, Comb. Sci. Tech. 179 (2007) 191-206.

[5] D. Veynante and L. Vervisch, Prog. Energy Combust. Sci. 28 (2002) 193-266.

[6] K. Bray, M. Champion, P. A. Libby, and N. Swaminathan, Combust. Flame 158 (2011) 2017-2022.

[7] Y. Gao, N. Chakraborty, and N. Swaminathan, Comb. Sci. Tech. 186 (2014) 13091337.

[8] Y. Gao, N. Chakraborty, and N. Swaminathan, J. Comb. 280671 (2014) 29.

[9] Y.-C. Chen and M. S. Mansour, Proc. Combust. Inst. 27 (1998) 811-818.

[10] F. T. C. Yuen and G. Ö.L. Proc. Combust. Inst. 34 (2013) 1393-1400.

[11] M. S. Sweeney, S. Hochgreb, M. J. Dunn, and R. S. Barlow, Combust. Flame 159 (2012) 2912-2929.

[12] Y. C. Chen and R. W. Bilger, Combust. Flame 131 (2002) 400-435.

[13] A. Buschmann, F. Dinkelacker, T. Schäfer, M. Schäfer, and J. Wolfrum, Proc. Combust. Inst. 26 (1996) 437-445.

[14] C. Kortschik, T. Plessing, and N. Peters, Combust. Flame 136 (2004) 43-50.

[15] C. Duwig, B. Li, Z. S. Li, and M. Aldén, Combust. Flame 159 (2012) 306-316.

[16] A. J. Aspden, M. S. Day, and J. B. Bell, J. Fluid Mech. 680 (2011) 287-320.

[17] A. Y. Poludnenko and E. S. Oran, Combust. Flame 157 (2010) 995-1011.

[18] S. A. Filatyev, J. F. Driscoll, C. D. Carter, and J. M. Donbar, Combust. Flame 141 (2005) 1-21.

[19] F. T. C. Yuen and Ö. L. Gülder, Proc. Combust. Inst. 34 (2013) 1393-1400.

[20] P. M. Allison, Y. Chen, M. Ihme, and J. F. Driscoll, Proc. Combust. Inst. 35 (2015) 3255-3262.

[21] B. Zhou, C. Brackmann, Q. Li, Z. Wang, P. Petersson, Z. Li, et al., Combust. Flame 162 (2015) 2937-2953.

[22] M. J. Dunn, A. R. Masri, R. W. Bilger, and R. S. Barlow, Flow Turbul. Combust. 85 (2010) 621-648.

[23] S. A. Kaiser and J. H. Frank, Proc. Combust. Inst. 32 (2009) 1639-1646.

[24] J. H. Frank and S. A. Kaiser, Exp. Fluids 44 (2008) 221-233. 
[25] G. H. Wang, R. S. Barlow, and N. T. Clemens, Proc. Combust. Inst. 31 (2007) 15251532.

[26] G. H. Wang, N. T. Clemens, R. S. Barlow, and P. L. Varghese, Meas. Sci. Technol. 18 (2007) 1287.

[27] M. S. Sweeney, S. Hochgreb, M. J. Dunn, and R. S. Barlow, Combust. Flame 159 (2012) 2896-2911.

[28] R. Zhou, S. Balusamy, M. S. Sweeney, R. S. Barlow, and S. Hochgreb, Combust. Flame 160 (2013) 2017-2028.

[29] N. Swaminathan and K. N. C. Bray, Combust. Flame 143 (2005) 549-565.

[30] H. Kolla, J. W. Rogerson, N. Chakraborty, and N. Swaminathan, Combust. Sci. Tech. 181 (2009) 518-535.

[31] S. A. Kaiser and J. H. Frank, Proc. Combust. Inst. 31 (2007) 1515-1523.

[32] M. Baum, T. J. Poinsot, D. C. Haworth, and N. Darabiha, J. Fluid Mech. 281 (1994) $1-32$.

[33] B. Gardiner, B. Yan, Z. Qin, G. Smith, D. Crosley, M. Golden, et al. GRI 3.0, available at <http://www.me.berkeley.edu/gri_mech/> .

[34] L. P. H. de Goey, T. Plessing, R. T. E. Hermanns, and N. Peters, Proc. Combust. Inst. 30 (2005) 859-866.

[35] K. N. C. Bray, Symp. Int. Combust. 17 (1979) 223-233. 


\section{Figure Captions}

Figure 1. Left half: schematic indicating FOV locations for distances above the burner surface. Streamlines and absolute velocity for SwB1 (left half) and SwB3 (right half). Rectangles represent the imaging locations [28].

Figure 2. An example of flame normal extracted from single shot profiles of OH-PLIF. (a) Instantaneous image of normalized OH LIF intensity, (b) temperature field (in K), overlaid with the flame front at the maximum gradient location (black line) and corresponding flame normal (white lines).

Figure 3. Single shot images of normalized OH LIF signal (top rows), temperature (middle rows) in $\mathrm{K}$, and temperature fluctuation, $T^{\prime}$, relative to the ensemble averaged temperature field, in K (bottom row) for no swirl (left) and high swirl (right). Top panel: FOV3, bottom: FOV1.

Figure 4: Mean (blue line) and rms (blue bars) fluctuation profiles of the temperature, for FOV1-3 along the normal coordinate $n$, where zero is the flame front. Black lines denote the unstrained laminar flame values for $\phi=0.75$.

Figure 5. Scatter plot of normalized angle (in multiples of $\pi$ ) between the normal directions for contours of temperature and $\mathrm{OH}$ as a function of the absolute value of the normalized distance from the flame front, coloured by a temperature index defined as $\Omega=\left(T-T_{o}\right) /\left(T_{f, 0}-T_{o}\right)$, where $T_{f, 0}$ represents the unstrained laminar flame temperature at the location of maximum gradient of $\mathrm{OH}$ at equivalence ratio of 0.75 . Magenta lines represent the mean and rms values. 
Figure 6. Instantaneous temperature fluctuation gradients for SwB1 and SwB3. Bottom to top: FOV1 to 3. The right hand side of the figure is the product side.

Figure 7. Conditioned probability density functions of $\lambda_{D}$ widths conditioned on temperature over a $20 \mathrm{~K}$ range.

Figure 8. Scatter plots of normalized Favre-averaged scalar dissipation rate, $\tilde{\chi}_{c}$, and mean Favre variance of progress variable temperature variance, $\widetilde{c}{ }^{\pi 2}$, colored by the corresponding mean local temperature. The SDR is normalized by the term $\left(\delta_{L} / S_{L}\right)$; where $S_{L}$ is the laminar flame speed of the methane/air flame $\left(S_{L}=0.214 \mathrm{~m} / \mathrm{s}\right)$ and $\delta_{L}$ is the laminar flame thickness $\left(\delta_{L}=0.588 \mathrm{~mm}\right)$ derived from laminar flame calculations at an equivalence ratio of $\phi=$ 0.75. The figures quoted on each panel are the slopes of each correlation. 


\section{Table Captions}

Table 1. Operating conditions. The velocity of the inner channel is $U_{i}=8.3 \mathrm{~m} / \mathrm{s}\left(\operatorname{Re}_{\mathrm{i}}=5960\right)$ and for the outer channel, $U_{o}=18.7 \mathrm{~m} / \mathrm{s},\left(\mathrm{Re}_{\mathrm{i}}=11500\right.$ based on hydraulic diameter of the outer annulus) with co-flow velocity $U_{c o}=0.4 \mathrm{~m} / \mathrm{s}$. Coordinates of the center of the FOV in radial and axial distances from the centreline at the base of the burner, in mm. SFR: ratio of incoming tangential to total flow rate.

Table 2. Maximum value of the ratio for the total mean velocity fluctuation $U^{\prime}$ divided by the laminar speed $S_{L}$ for a given axial location, $z$. Turbulent velocity measurements acquired with laser Doppler anemometry (LDA) and particle image velocimetry (PIV) by Zhou et al. [28]. 


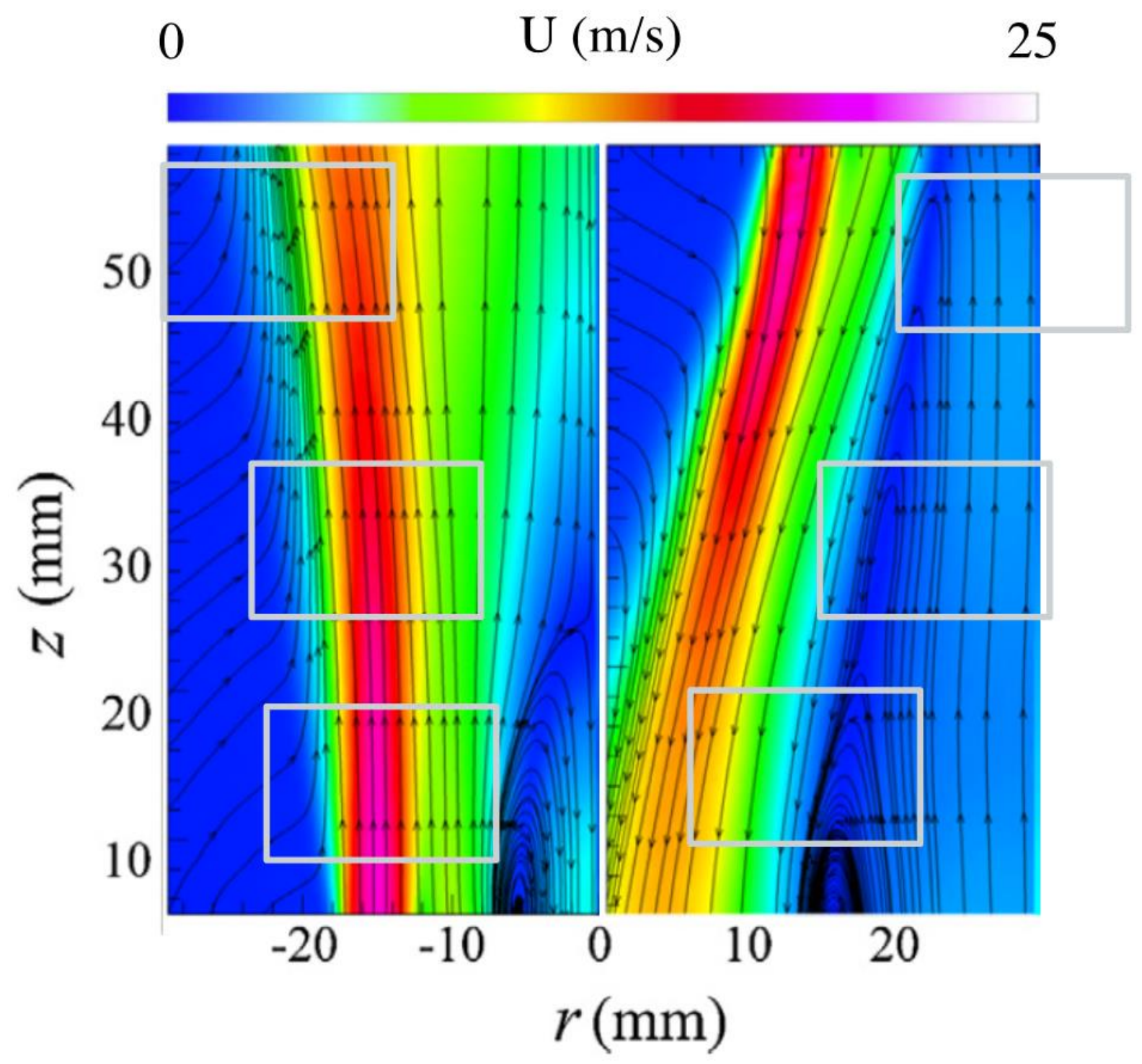

Figure 1 
(a) OH-PLIF

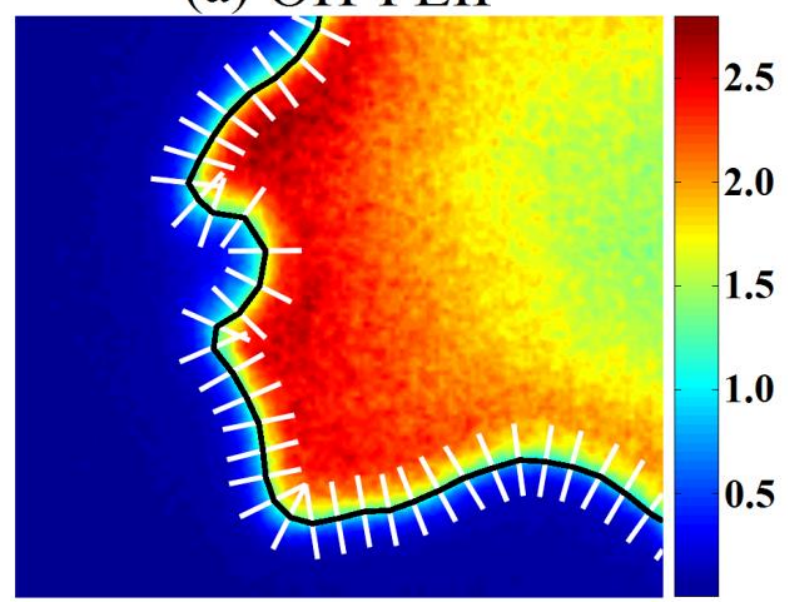

Figure 2 (b) $T$

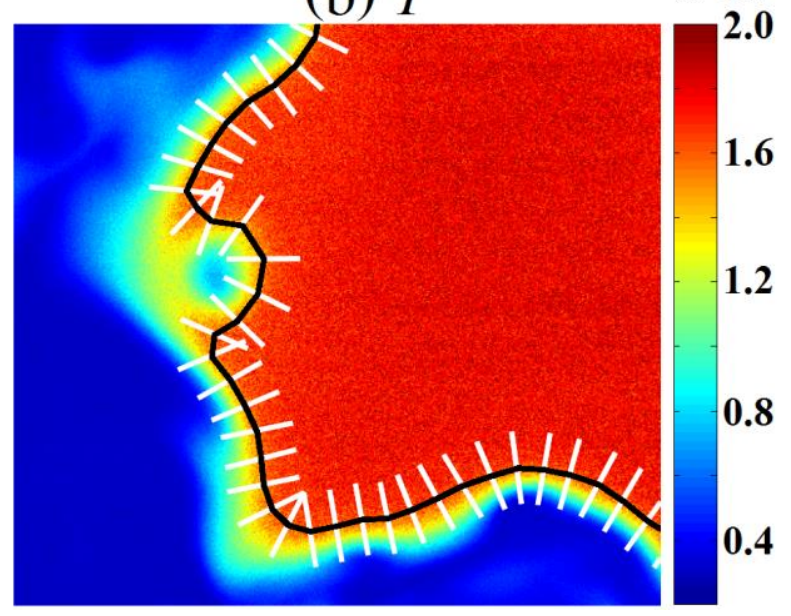



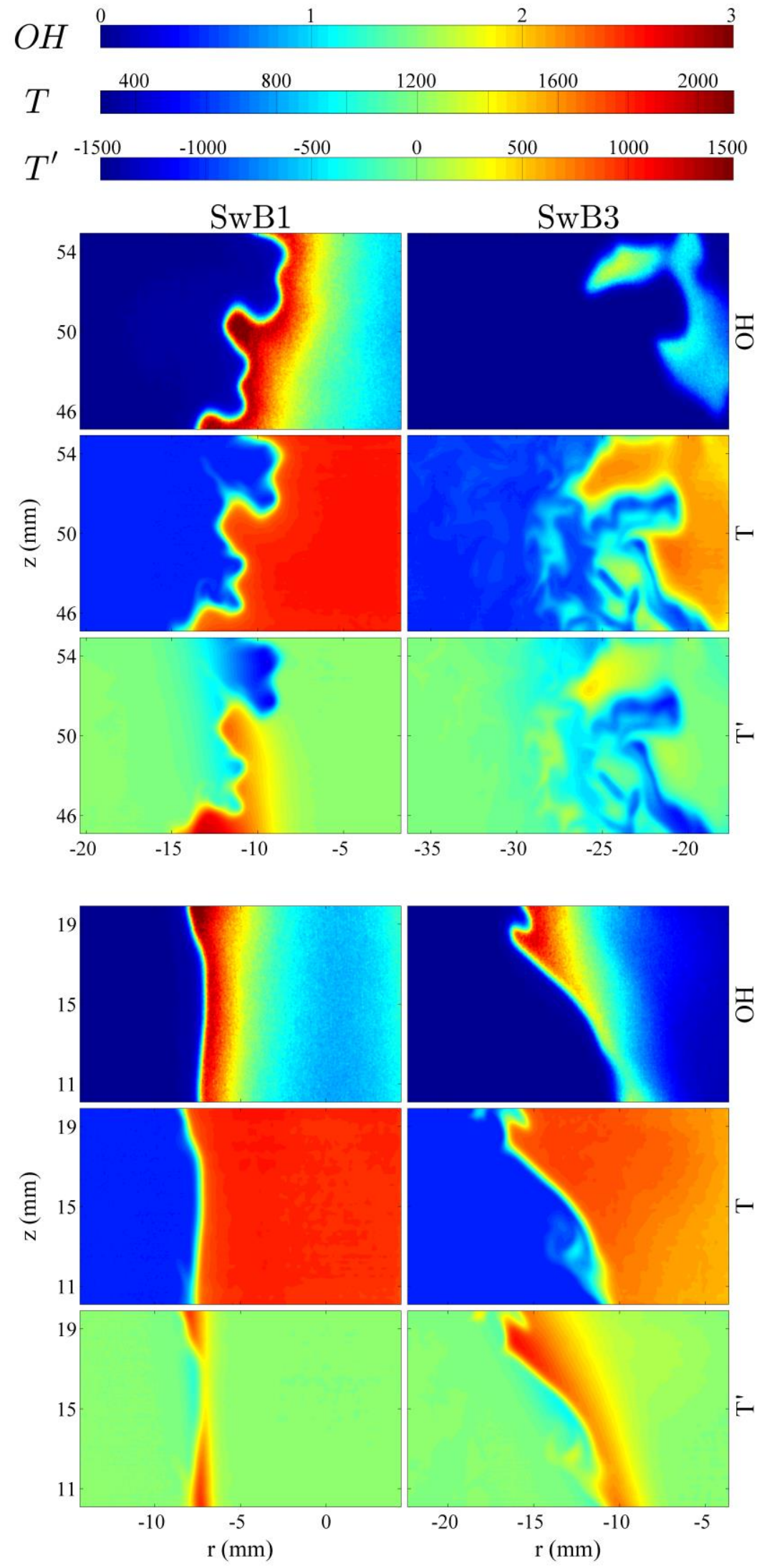

Figure 3 


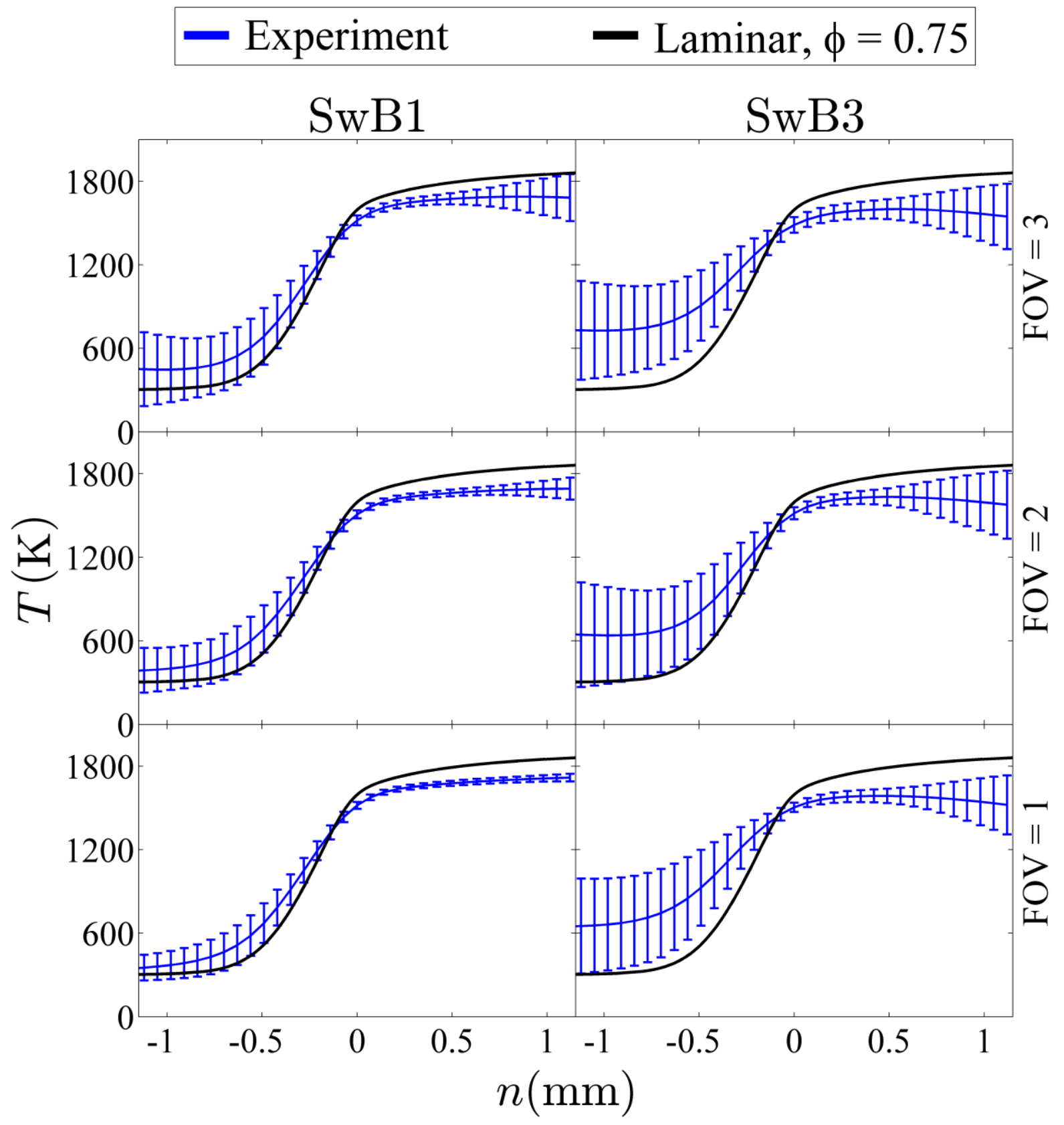

Figure 4 


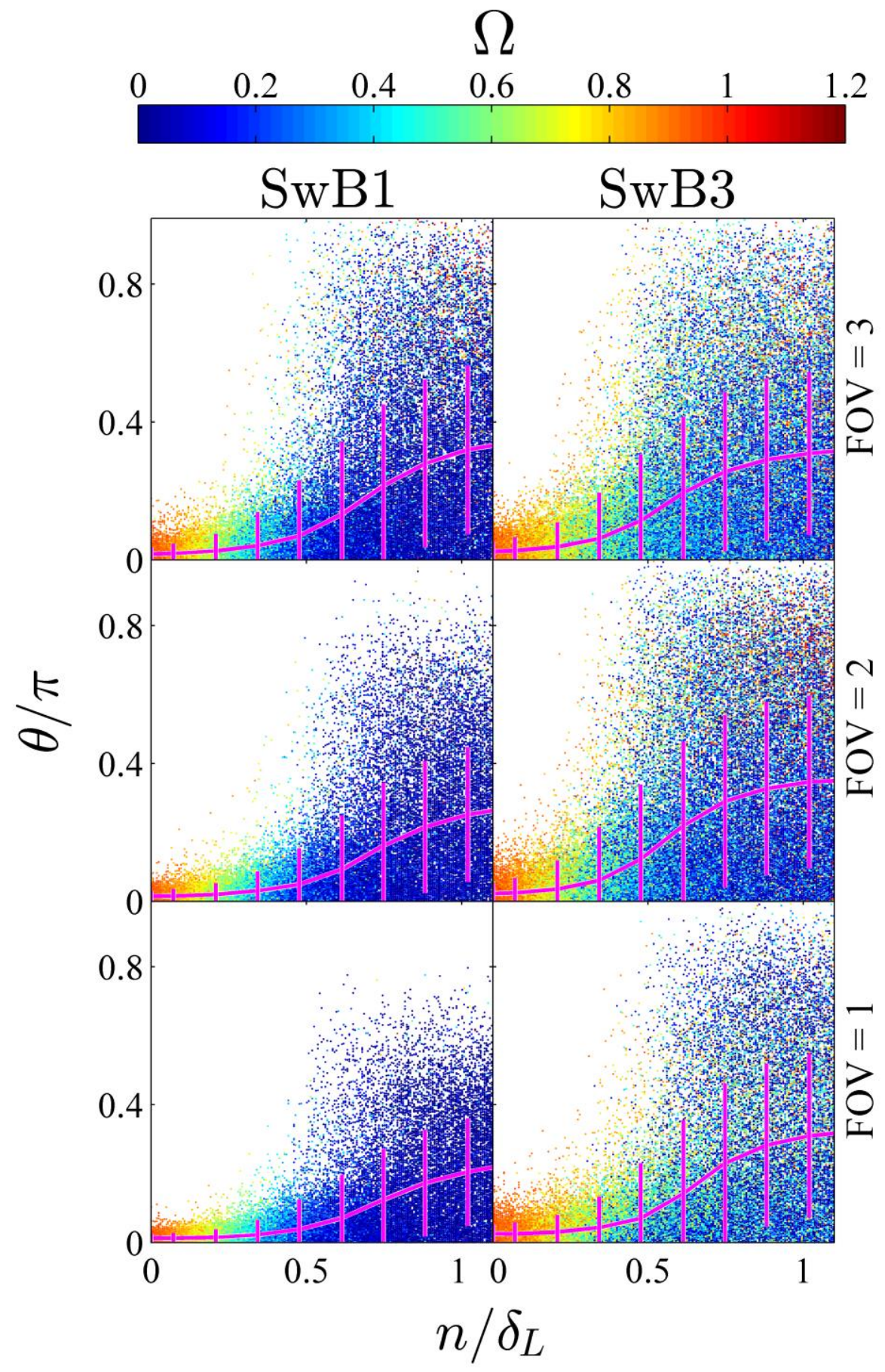

Figure 5 


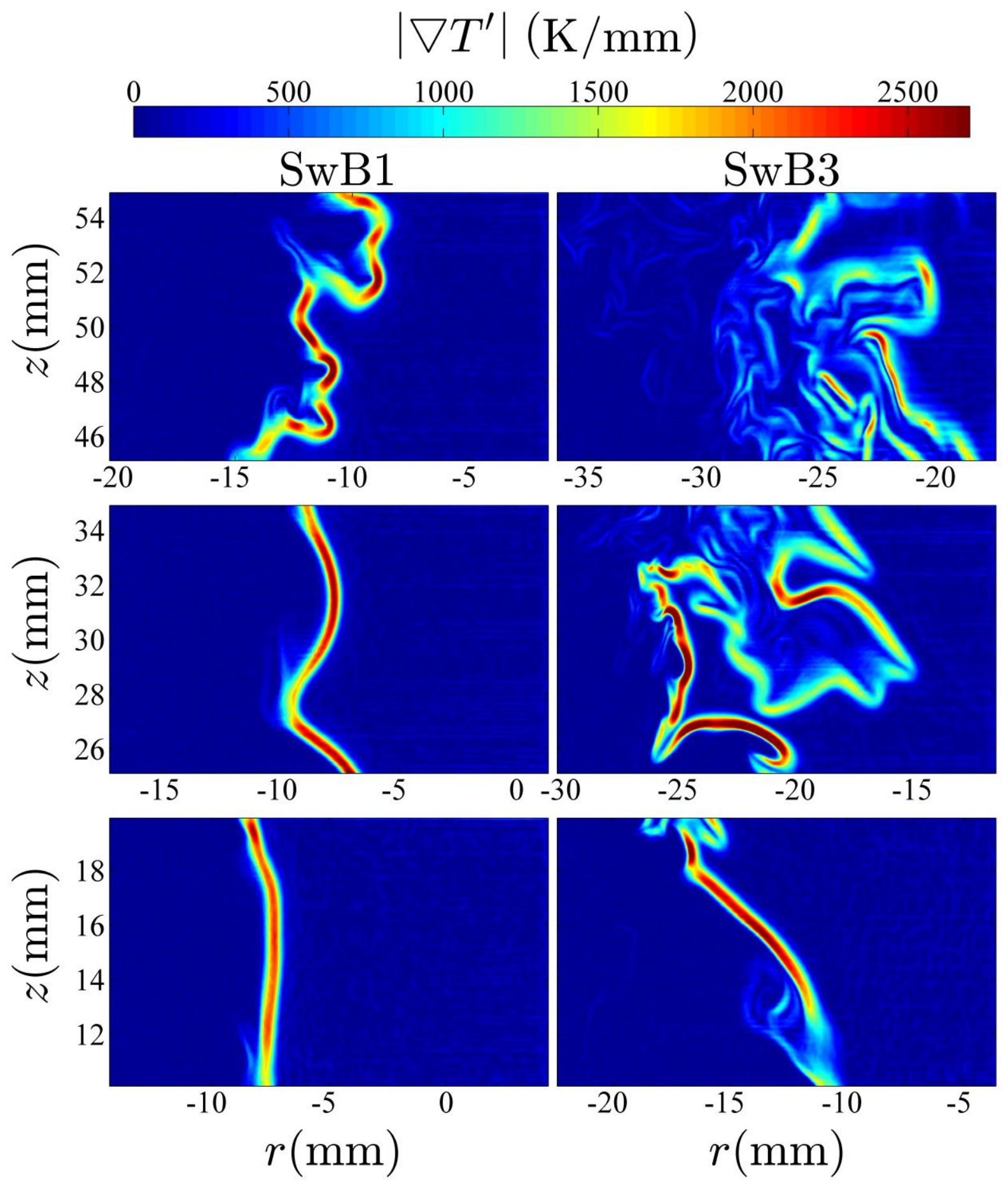

Figure 6 


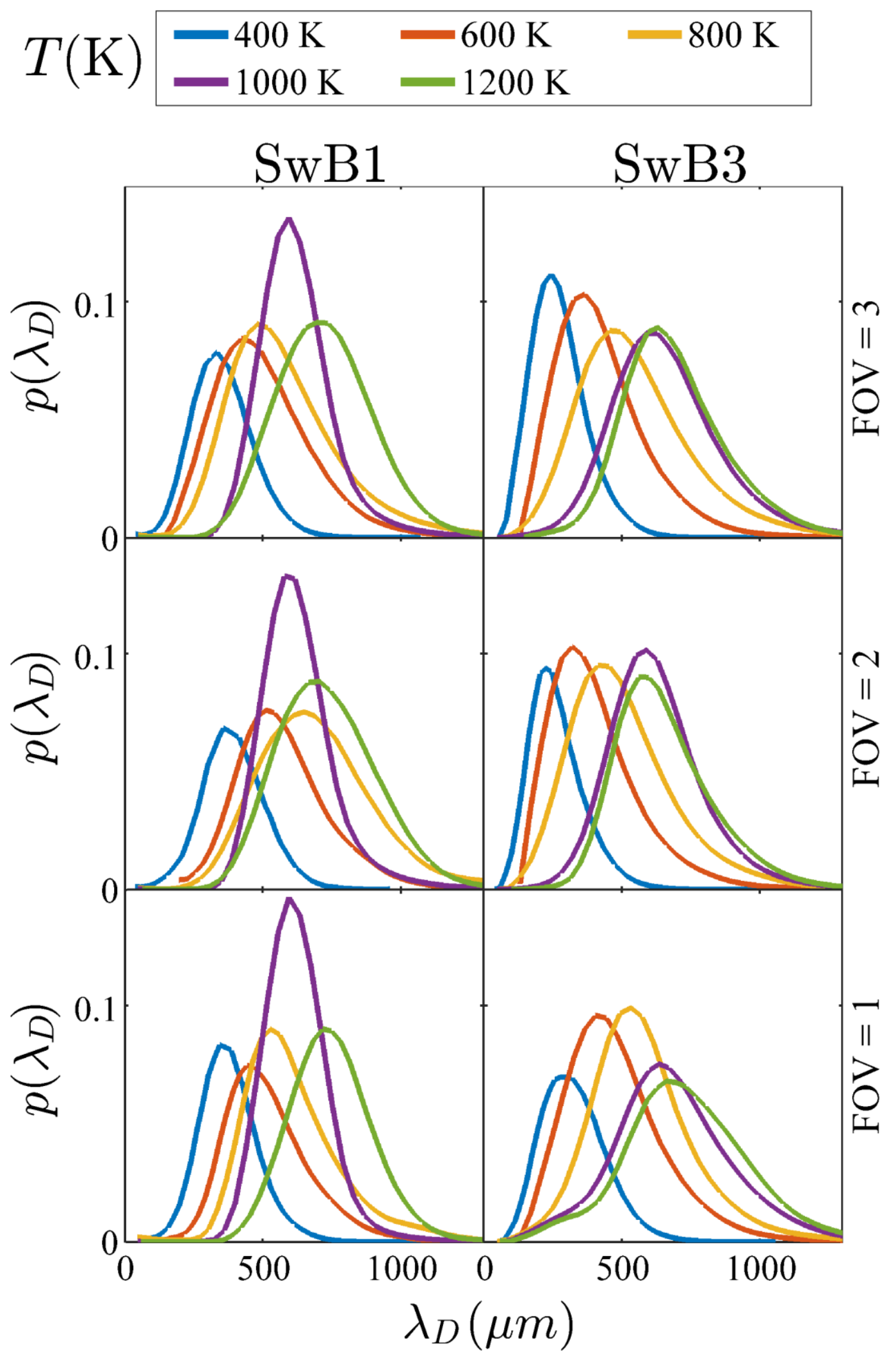

Figure 7 


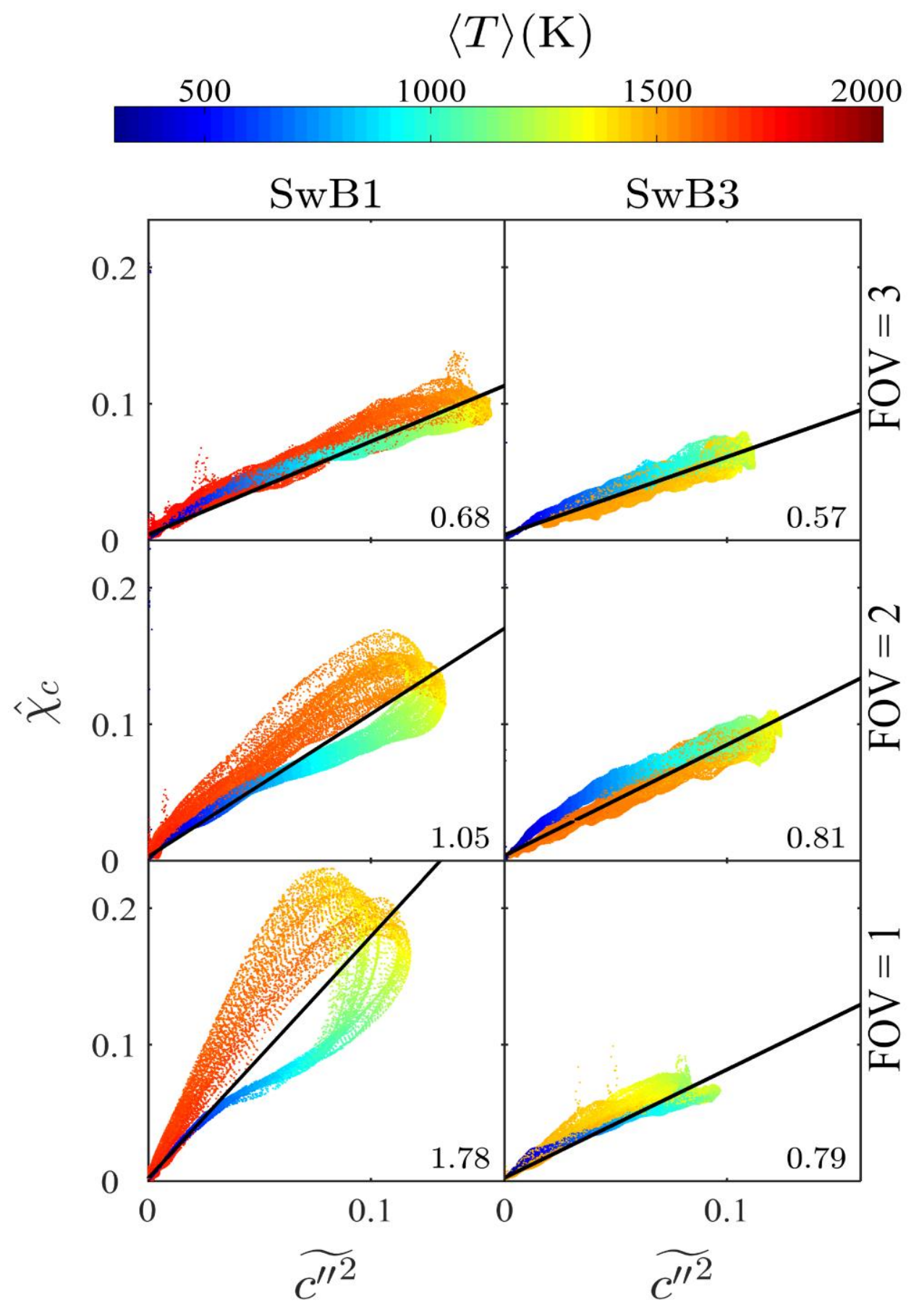

Figure 8 